\title{
TOP-QUARK DIFFERENTIAL CROSS-SECTION MEASUREMENTS WITH THE ATLAS DETECTOR*
}

\author{
Matteo Scornajenghi \\ on behalf of the ATLAS Collaboration \\ INFN Gruppo Collegato di Cosenza and Università della Calabria, Italy
}

(Received April 20, 2018)

The most recent results on top-quark pairs and single top-quark differential cross-section measurements in proton-proton $(p p)$ collisions with the ATLAS detector at the Large Hadron Collider (LHC) at $\sqrt{s}=8$ and $13 \mathrm{TeV}$ are presented. The results are compared to the latest QCD theoretical calculations.

DOI:10.5506/APhysPolB.49.1353

\section{Introduction}

The top quark was discovered by both the CDF [1] and the $\mathrm{D} \emptyset[2]$ collaborations in 1995 at the Tevatron Collider. It is the most massive elementary particle known and the heaviest quark of the Standard Model (SM). The top-quark physics provides a sensitive probe to investigate the Higgs boson properties, to test the validity of the SM and to potentially discover physics beyond the SM, since its properties are precisely predicted by the theory. In this overview, some of the most recent measurements of top-quark pair $(t \bar{t})$ and single top-quark differential cross sections performed by the ATLAS Collaboration using data collected in $2012\left(\mathcal{L}=20.2 \mathrm{fb}^{-1}\right.$ at $\left.\sqrt{s}=8 \mathrm{TeV}\right)$, $2015\left(\mathcal{L}=3.2 \mathrm{fb}^{-1}\right.$ at $\left.\sqrt{s}=13 \mathrm{TeV}\right)$ and $2015+2016\left(\mathcal{L}=36.1 \mathrm{fb}^{-1}\right.$ at $\sqrt{s}=13 \mathrm{TeV})$ are presented. The ATLAS detector and its performance are described in [3].

\footnotetext{
* Presented at the Cracow Epiphany Conference on Advances in Heavy Flavour Physics,
} Kraków, Poland, January 9-12, 2018. 


\section{Single top-quark differential cross-section measurements at $\sqrt{s}=8 \mathrm{TeV}$ with the ATLAS detector in the $t$-channel}

Single top quarks are produced in $p p$ collisions via electroweak chargedcurrent interactions. At Leading Order (LO), three different processes may take place, according to the virtuality of the exchanged $W$ boson: timelike ( $t$-channel), space-like ( $s$-channel) and the associated production of a single top quark and an on-shell $W$ boson (Wt-channel). At the LHC, the $t$-channel mechanism is the dominant one, followed by the $W t$ associated production and the $s$-channel. At $\sqrt{s}=8 \mathrm{TeV}$, ATLAS has performed differential cross-section measurements in the $t$-channel using the lepton + jets $(\ell+$ jets $)$ signal topology [4]. The top quark is reconstructed by means of the Pseudo-Top algorithm [5], which builds the top-quark four-momentum from its decaying products and the missing transverse momentum $\left(\vec{E}_{\mathrm{T}}^{\mathrm{miss}}\right)$. Since the resulting system is underconstrained, the mass of the $W$ boson to which the top-quark decays is fixed. To separate $t$-channel single quark signal events from the expected background, a high $E_{\mathrm{T}}^{\text {miss }}$ is required due to the presence of a neutrino, as well as a $b$-tagged jet (coming from the $W t b$ vertex). In addition, several kinematic variables have been combined into one discriminant by employing a neural network (NN) discriminant, trained on simulated events. QCD multi-jet background is extracted via a data-driven technique, while the other backgrounds are estimated via Monte Carlo simulation. The spectra are then corrected for detector effects by means of a Bayesian iterative unfolding technique [7]. The same technique is adopted for the other analyses presented. Figure 1 shows the measured cross sections

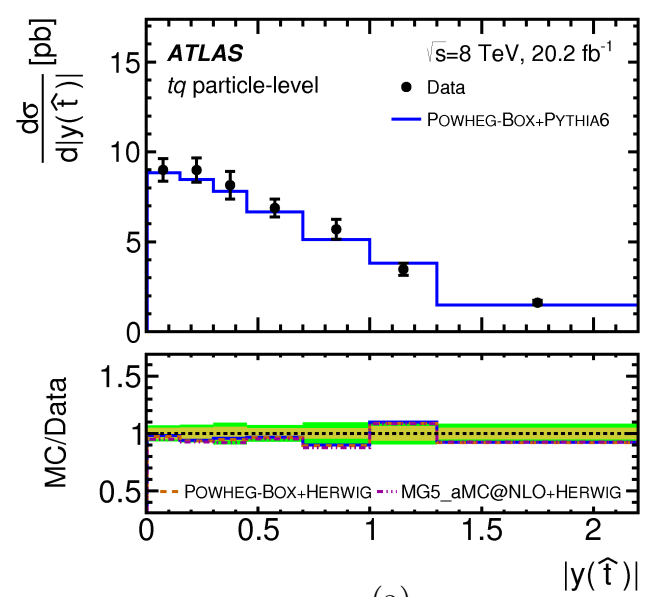

(a)
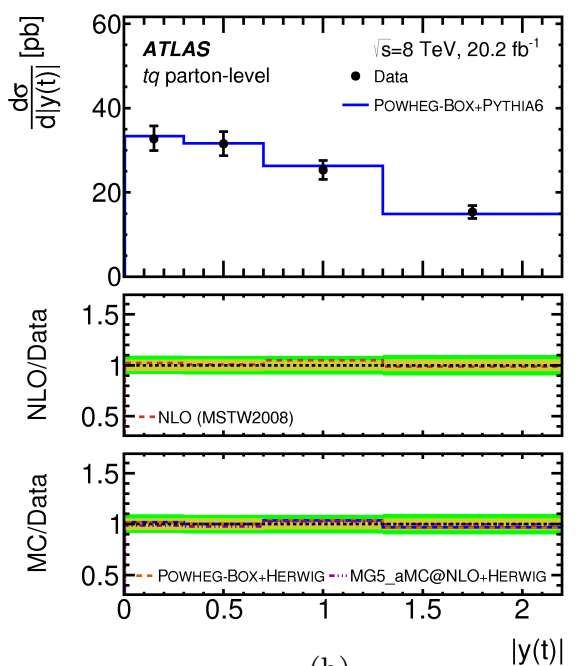

(b)

Fig. 1. Absolute differential cross sections as a function of the absolute value of the top-quark rapidity at particle (a) and parton (b) levels [4]. 
as a function of the absolute value of the top-quark rapidity both at particle level and parton level. The measured spectra are compared with different Monte Carlo (MC) generators (Powheg and MG5_aMC@NLO, showered with Herwig) and, at parton level, with a next-to-leading order perturbative QCD prediction. The SM predictions provide a good description of data.

\section{Single top-quark differential cross-section measurements at $\sqrt{s}=13 \mathrm{TeV}$ with the ATLAS detector in the $W t$-channel}

With the large set of data collected with the ATLAS detector during the 2015 and 2016 campaigns $\left(\mathcal{L}=36.1 \mathrm{fb}^{-1}\right)$ at a center-of-mass energy of $\sqrt{s}=13 \mathrm{TeV}$, the ATLAS Collaboration was able to perform differential cross-section measurements also in the $W t$ channel in the oppositecharge sign dilepton decay topology [6], which is the purest one. The largest source of background comes from $t \bar{t}$ production events. The background suppression is achieved by requiring exactly one $b$-tagged jet in the final state and applying a Boosted Decision Tree (BDT) discriminant. Since the top quark is kinematically underconstrained, no top-quark reconstruction is performed. Figure 2 shows the measured absolute differential cross section as a function of the energy of the $b$-tagged jet, compared with Powheg+Pythia6, Powheg+Herwig++ and MG5_aMC@NLO+Herwig++ MC predictions. A good agreement is observed in the bulk of the distribution.

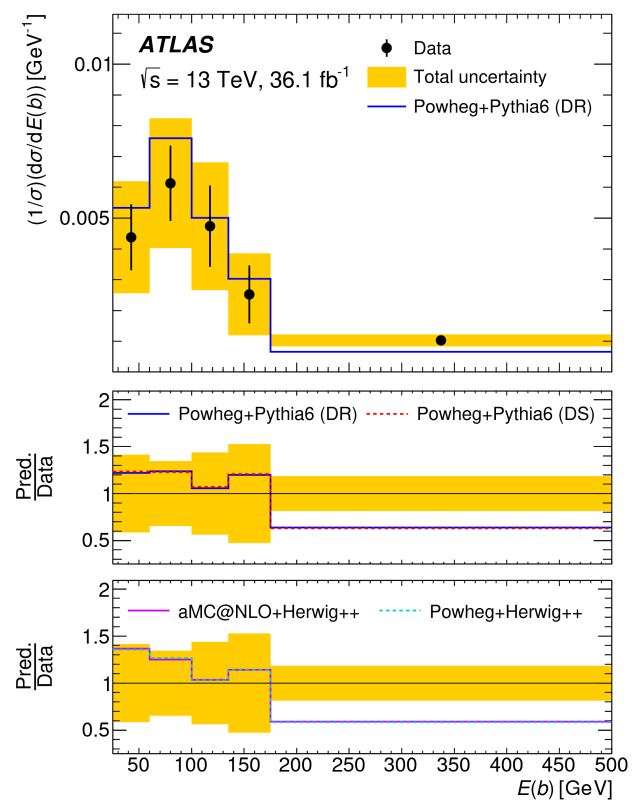

Fig. 2. Absolute differential cross sections as a function of the energy of the $b$-tagged jet at particle level [6]. 


\section{4. $t \bar{t}$ production differential cross-section measurements at $\sqrt{s}=13 \mathrm{TeV}$ with the ATLAS detector}

At LO, the $t \bar{t}$ production originates from two production modes: $q \bar{q}$ annihilation and gluon-gluon ( $g g$ ) fusion. At the LHC and for $\sqrt{s}=13 \mathrm{TeV}$, the $g g$ fusion process dominates, accounting for almost the $90 \%$ of the total production rate. The top quark decays almost exclusively into a $W$ boson and a bottom quark. The $t \bar{t}$ events are thus classified according to the decay modes of the two $W$ bosons. When both $W$ bosons decay hadronically, the event is defined fully hadronic. This is the channel with the highest branching ratio $(\mathrm{BR} \sim 44 \%)$, but it also has the largest background contamination. When the two $W$ bosons decay leptonically, the channel decay mode is called dilepton, which is the purest one, but with the lowest branching ratio ( $\mathrm{BR} \sim 10 \%$ ). When one of the two bosons decays hadronically and the other one decays leptonically, the channel is defined $\ell+$ jets. It is also referred to as a golden channel, since it is the best compromise between branching ratio $(\mathrm{BR} \sim 46 \%)$ and background contamination. The measurements of the $t \bar{t}$ production differential cross sections allow to perform stringent tests of the SM, since the theoretical predictions are available at the next-to-next-leading order (NNLO). This process also constitutes the main background for Beyond Standard Model (BSM) searches and it is also sensitive to gluon PDFs. The ATLAS Collaboration performed differential cross-section measurements of the $t \bar{t}$ system in all the three different channels at $\sqrt{s}=13 \mathrm{TeV}$ using data collected during the 2015 [8,9] and 2015+2016 campaigns [11]. Due to the substantial differences between the channels, distinct approaches have been employed to reconstruct the $t \bar{t}$ system and improve the selection efficiency, while reducing the background contamination. In the $\ell+$ jets channel, two different regimes are taken into account, defined by the $p_{\mathrm{T}}$ of the hadronically decaying top quark. When the top quark has a low $p_{\mathrm{T}}$, its decay products are well-resolved in space (hence it is referred to as resolved regime), whilst the decay products of an high- $p_{\mathrm{T}}$ top quark will be subjected to a Lorentz boost and will be revealed by the detector as a large radius jet. This regime is also known as boosted regime. The multijet background is estimated through a data-driven matrix method techniques, the $W$ production in association to jets is estimated by a mixture of MC-based and data-driven techniques, while the other sources of background are MC-based only. The system reconstruction, for the resolved regime, is handled through the Pseudo-Top algorithm, whilst, in the boosted regime, the highest- $p_{\mathrm{T}}$ top-tagged large- $R$ jet is identified as the hadronic top quark. Figure 3 (a) shows the measured relative differential cross section as a function of the hadronic top $p_{\mathrm{T}}$ compared to several MC predictions; some tension between data and most predictions is observed. In the dilepton channel, events presenting opposite-sign $e \mu$ leptons are selected. This is the 
purest channel, since the contamination from $Z$ production is strongly suppressed. The background estimation is fully estimated using Monte Carlo predictions. The largest expected source of background is from $W t$ associated production. The system reconstruction is performed via the neutrino weighting algorithm [10]. It consists of spanning the $\left(\eta_{\nu}, \eta_{\bar{\nu}}\right)$ plane, being $\eta_{\nu}$ $\left(\eta_{\bar{\nu}}\right)$ the pseudorapidity of the final-state (anti)neutrino. For each $\left(\eta_{\nu}, \eta_{\bar{\nu}}\right)$ pair, the resulting $E_{\mathrm{T}}^{\text {miss }}$ is compared with the measured one and a Gaussian weight is estimated. The pair corresponding to the smallest weight is taken as the correct one. Figure 3 (b) shows the relative measured cross section for the top $p_{\mathrm{T}}$ spectra compared with different MC predictions. Most

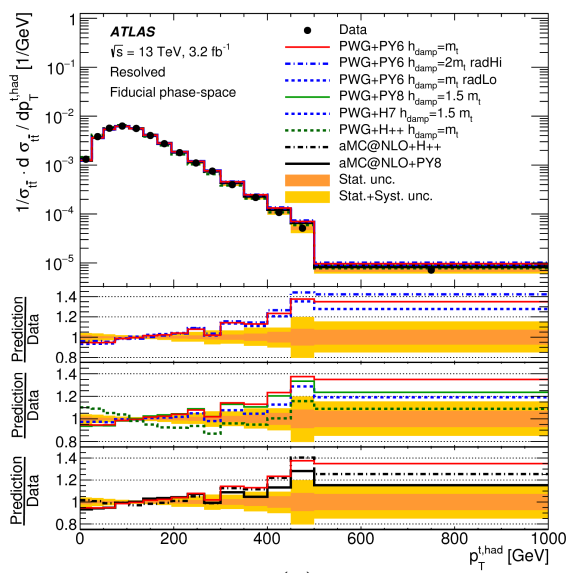

(a)

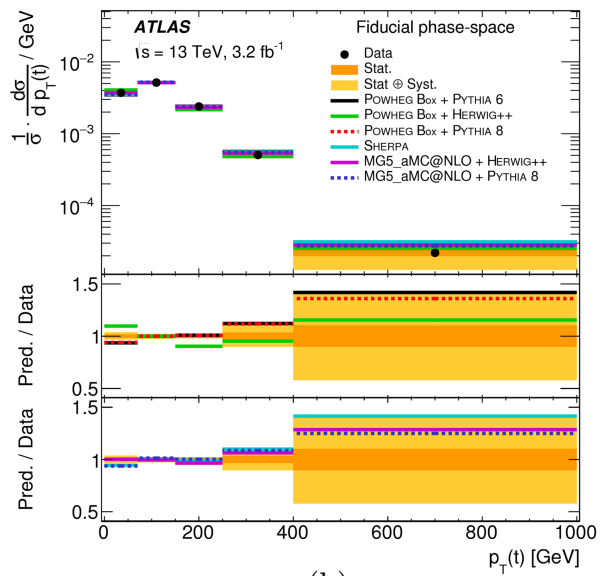

(b)

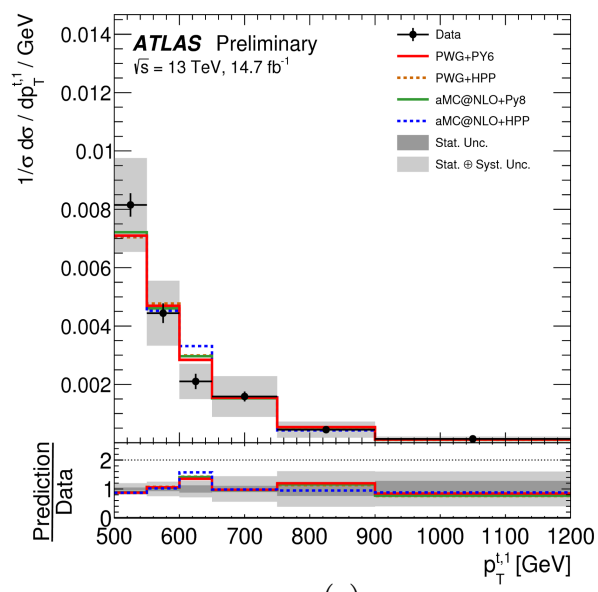

(c)

Fig. 3. Relative differential cross section as a function of the $p_{\mathrm{T}}$ of the hadronic top quark in the resolved regime (a) in the $\ell+$ jets channel [8], of the top quarks and antiquarks in the dilepton channel (b) [9] and of the leading- $p_{\mathrm{T}}$ top quark in the fully hadronic channel (c) at particle level [11]. 
generators agree with the unfolded data within the experimental uncertainties, with the exception of the Powheg+Herwig++ MC simulation. Both the measurements in the $\ell+$ jets and the dilepton channels have been performed using the full 2015 dataset collected with the ATLAS detector. Finally, the measurement in the fully hadronic channel, performed with the full 2015 and 2016 dataset in the di-boosted regime is presented. The events are required to present two top-tagged high- $p_{\mathrm{T}}$ large- $R$ jets. Both of them are required to be associated to a small- $R$ b-tagged jet. The dominant source of background comes from QCD multi-jets events that are estimated with a data-driven technique. The other sources of background are based on MC predictions. Figure 3 (c) shows the measured relative spectra for the leading top $p_{\mathrm{T}}$, compared with MC. The several predictions agree with data within uncertainties.

\section{Conclusions}

The latest top quark and $t \bar{t}$ production differential cross-section measurements performed by the ATLAS Collaboration exploiting data collected by the ATLAS detector at the LHC at a center-of-mass energy of 8 and $13 \mathrm{TeV}$ have been presented. The results shown are, in general, in a good agreement with latest SM predictions. It has been shown that the ATLAS Collaboration, by performing high-precision measurements, is able to conduct stringent tests of the SM and, concerning the $t \bar{t}$ measurements, it can explore very high- $p_{\mathrm{T}}$ phase-space regions and obtain results that are consistent between different channels.

\section{REFERENCES}

[1] CDF Collaboration, Phys. Rev. Lett. 74, 2626 (1995) [arXiv:hep-ex/9503002].

[2] D $\emptyset$ Collaboration, Phys. Rev. Lett. 74, 2632 (1995) [arXiv: hep-ex/9503003].

[3] ATLAS Collaboration, JINST 3, S08003 (2008).

[4] ATLAS Collaboration, Eur. Phys. J. C 77, 531 (2017).

[5] ATLAS Collaboration, J. High Energy Phys. 1506, 100 (2015).

[6] ATLAS Collaboration, Eur. Phys. J. C 78, 186 (2018) [arXiv:1712.01602 [hep-ex]].

[7] G. D'Agostini, Nucl. Instrum. Methods Phys. Res. A 362, 487 (1995).

[8] ATLAS Collaboration, J. High Energy Phys. 1711, 191 (2017).

[9] ATLAS Collaboration, Eur. Phys. J. C 77, 292 (2017).

[10] D $\emptyset$ Collaboration, Phys. Rev. Lett. 80, 2063 (1998).

[11] ATLAS Collaboration, arXiv:1801.02052 [hep-ex] submitted to Phys. Rev. D. 